\title{
Myasthenia gravis: long-term prognostic value of thymus lactate dehydrogenase isoenzyme pattern of hyperplastic thymus and thymoma
}

\author{
ISTVÁN SZATHMÁRY, LÁSZLÓ SELMECI, ELEK PÓSCH, ALBERT SZOBOR, \\ JÁNOS MOLNÁR
}

From the Departments of Neurology and Surgery, Institute of Pulmonology, Hospital of the Hungarian State Railway; Institute of Pathophysiology, Semmelweis University Medical School; Department of Neurology, Jahn Ferenc University Teaching Hospital, Budapest, Hungary

SUMMARY Lactate dehydrogenase (LDH) isoenzyme pattern and the percent of $\mathrm{H}$-subunit content were determined in the thymus of 62 patients ( 55 with hyperplasia, 7 with tumours) after thymectomy. An increase in $\mathrm{LDH}_{1}$ relative activity indicates that in the thymus of patients with myasthenia gravis the ratio of mature differentiated thymocytes was higher than in the thymus of control subjects. LDH isoenzyme profiles of thymus tumours were similar to those described in other neoplasms, except that thymomas with apparent predominance of epithelial cells and with minimal lymphocytic reaction exhibited a marked elevation only in $\mathrm{LDH}_{2}$ relative activity, presumably associated with the specific (secretory) function of epithelial cells. The elevation of $\mathrm{H}$-subunit content, a parameter characteristic of both thymic components (lymphoid and epithelial), correlated closely with a poor clinical condition in patients several years after surgery.

Several data ${ }^{1-4}$ indicate an autoimmune aetiology for myasthenia gravis. It is of particular interest that the thymus is a central organ of specific immune responses and that the autoimmune processes in myasthenia gravis are initiated in this gland. The immune status of the human thymus has been the subject of many investigations, but all the methods used have been immunological, that is based on the antigen-antibody reaction. In autoimmune disorders these techniques have disadvantages. During the course of disease the majority of antibodies are bound either to the cells of the target organ or are in the form of immunocomplexes, so that the surface of the immunoreactive cells under study may be covered by specific antigens.

There are available, however, other methods which utilise intracellular enzymic markers ${ }^{56}$ characteristic of the maturation and differentiation of immunocompetent cells. According to Plum et al ${ }^{7}$

\footnotetext{
Address for reprint requests: Dr I Szathmáry, Institute of Pulmonology, Hospital of the Hungarian Railway, Szanatórium Str $2 / a$, Budapest, Hungary H-1528.
}

Received 8 May 1984 and in revised form 30 November 1984 Accepted 2 February 1985 lactate dehydrogenase (LDH) isoenzyme patterns can not only differentiate between $T-$ and B-lymphocytes but also can follow the course of maturation of the whole T-cell line. ${ }^{8}$ In haematological practice this method is known as a lymphocytic marker but recently it has been used in autoimmune disorders, ${ }^{10}$ too. In myasthenia gravis no such studies have been reported and data using traditional immunological techniques are scarce for long-term follow-up studies of similar duration (mean 6.5 years) as in this paper.

\section{Patients and methods}

Thymus samples obtained through thymectomy from 62 patiens (48 females, 14 males) with myasthenia gravis were studied. Patients' mean age was 29 years (range 15-54 years). In 39 instances the disease exhibited a slow course whereas in 23 cases rapid progression was noted. In 55 patients the disease was generalised, in one it was oculoskeletal and in another bulbo-skeletal; in three subjects there was skeletal involvement alone, and in two the localisation was purely bulbar. The mean duration of the disease (that is the period from the onset of disease to the study of the removed thymus) was 4.7 years (range 4 months -20 years). The mean duration of the follow-up after operation was 6.5 years (range $5-8.5$ years). At vari- 


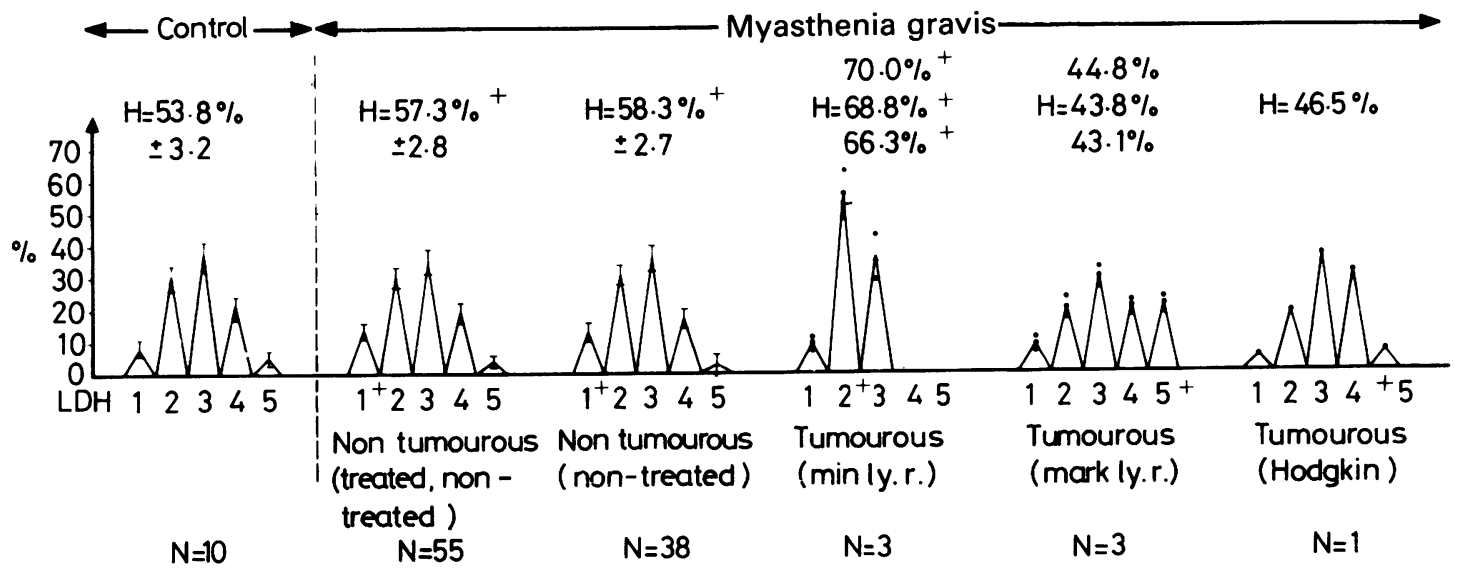

Fig 1 Lactate dehydrogenase $(\mathrm{LDH})$ isoenzyme pattern and percent of $H$-subunit content in the thymus of patients with myasthenia gravis $\bar{x} \pm S D$. "significant difference from controls $(p<0.001)$. "Not treated" means that the patients had not received $x$-irradiation and/or azathioprine treatment within two years prior to studies. "min.ly.r." = minimal lymphocytic reaction. "mark.ly.r." = marked lymphocytic reaction. "Hodgkin" = Hodgkin-type lymphoma

ous times prior to surgery 25 patients had been subjected to mediastinal X-ray therapy (total dose delivered in three weeks was $5000 \mathrm{r}=15 \times 174 \mathrm{cGY}$ under conditions for deep irradiation) and/or to immunosuppressive treatment (100 mg azathioprine per os administered daily for 90 days). Since these treatments might influence LDH isoezyme distribution, 17 patients who had received treatment within two years prior to examinations were excluded from this study. Steroid or other treatments affecting the immune system were not administered, but all patients received anticholinesterase or adjuvant therapy.

The thymus was removed via median sternotomy. Samples were taken from the medulla and prepared for light microscopic examinations using routine histological techniques and haematoxylin-eosin staining. Out of seven patients with thymus tumours six had thymomas and in one case Hodgkin-type lymphoma was diagnosed. Of the six thymoma cases, minimal (three cases) or marked (three cases) lymphocytic reactions were noted. ${ }^{11}$ Control thymus samples derived from 10 patients ( 7 females, 3 males) with mean age of 34 years (range 18-53 years) underwent surgery for substernal goitre, diaphragmatic hernia or bullous emphysema.

LDH isoenzymes were separated by agar-gel electrophoresis $^{12}$ in aliquots of supernatant of the freshly homogenised (phosphate buffer $0.05 \mathrm{M}, \mathrm{pH} 7.4$ ) thymus sample and visualised according to the method described by Van der Helm. ${ }^{13}$ The isoenzyme bands were scanned in a densitometer (Kipp and Zonen, Holland) and their relative activities were expressed as a percentage. In the knowledge of the percent distribution of the LDH isoenzymes, the proportion of $\mathbf{H}$ and $M$ subunits was also calculated in percent. ${ }^{14}$ Structural difference among the five LDH isoenzymes with similar function is caused by their different subunit ( $\mathrm{H}$ and $M$ ) compositions; $\mathrm{LDH}_{1}=\mathrm{H}_{4}$, $\mathrm{LDH}_{2}=\mathrm{H}_{3} \mathrm{M}_{1}, \mathrm{LDH}_{3}=\mathrm{H}_{2} \mathrm{M}_{2}, \mathrm{LDH}_{4}=\mathrm{H}_{1} \mathrm{M}_{3}, \mathrm{LDH}_{5}=$ $\mathbf{M}_{\mathbf{4}}$.
Data were analysed for statistical significance using Student's paired or unpaired $t$ tests, single variance analysis, linear regression and correlation analysis.

\section{Results}

Data in fig 1 indicate that in the group of 55 nontumour patients including 38 myasthenia gravis patients with no treatment the relative activity of $\mathrm{LDH}_{1}$ isoenzyme in the thymus was significantly ( $\mathrm{p}$ $<0.001)$ higher $(13.0 \pm 2.2 \%$ and $12.9 \pm 2.7 \%)$ than that in control thymus $(7 \cdot 7 \pm 2 \cdot 3 \%)$. Although the proportion of $\mathrm{H}$-subunit $(57 \cdot 3 \pm 2 \cdot 8 \%$ and $58 \cdot 3$ $\pm 2.7 \%$ ) was higher in both groups than in control subjects $(53.8 \pm 3.2 \%)$ this difference may not have biological importance. On the other hand, in the 38 myasthenia gravis patients with no tumour and no treatment there was a close correlation between $\mathrm{H}$-subunit ratio and duration of disease $(\mathrm{r}=0.8803$, $p<0.001)$ and the clinical condition at 6.5 years after operation $(r=0.8594, p<0.001)$, although the range $(54 \cdot 2-63 \cdot 2 \%)$ was narrow (fig 2$)$. Clinical condition was graded by points from 0 to 10 as described by Szobor ${ }^{15}$ where severe forms received higher scores.

Out of the seven tumour patients in three instances the thymomas were associated with minimal lymphocytic reactions. In these subjects' tumour the LDH isoenzyme pattern was markedly shifted with predominance of $\mathrm{LDH}_{2}(62.2,55.3$ and $48.5 \%)$ and $\mathrm{H}$ subunit as compared to control $\mathrm{LDH}_{2}$ (29.9 $\pm 3.7 \%$ ), whereas $\mathrm{LDH}_{4,5}$ were not detectable (fig 1). Both changes were significant 

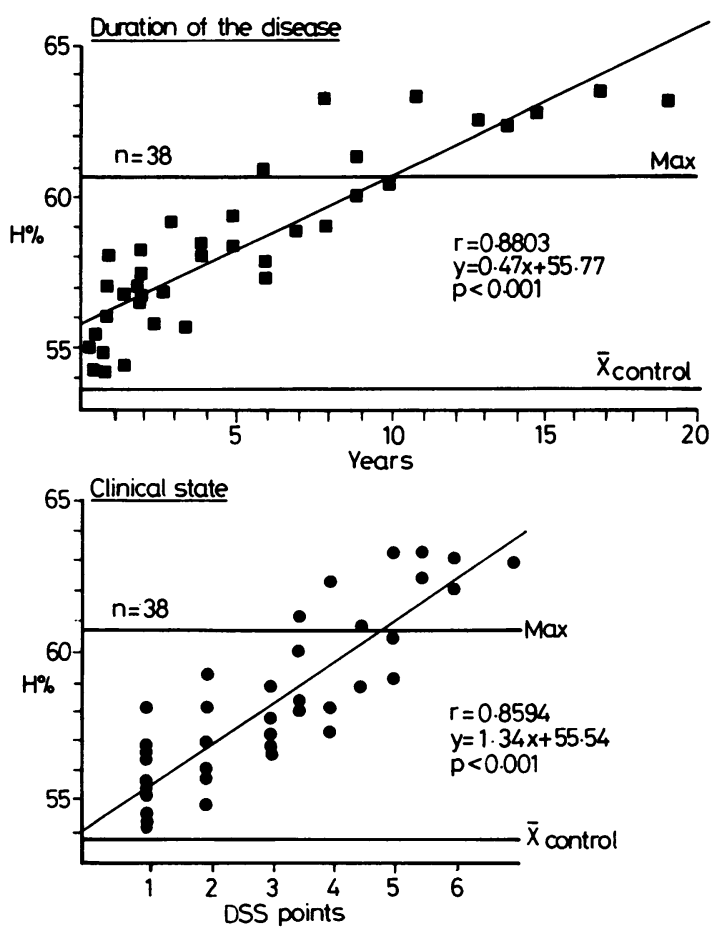

Fig 2 Correlation between percent of $\mathrm{H}$-subunit content in the thymus and duration of disease (upper panel) and between clinical condition at various times (mean: 6.5 years, range 5-8 years) after surgery (lower panel). Thymus samples were removed from non-tumour-patients with myasthenia gravis who had not received azathioprine and/or $x$-irradiation treatments within two years. "DSS": score for patients' clinical conditions. ${ }^{15}$ Higher values correspond to poor condition. $\bar{X}$ contr.: control mean \pm range maximum and minimum values.

( $p<0.001)$ either compared with control or with values of myasthenia gravis patients with no tumour. In all of the three thymoma cases with enhanced lymphocytic reaction $\mathrm{LDH}$ isoenzyme profiles were shifted with prominence of $\mathrm{LDH}_{5}(18 \cdot 8,22 \cdot 2$, and $21 \cdot 1 \%)$ compared with control $\mathrm{LDH}_{5}(4 \cdot 8 \pm 2 \cdot 2 \%)$, whereas the contribution of $\mathrm{LDH}_{2}$ was decreased in association with a reduction in the ratio of H-subunit. Both changes were significant statistically ( $p<0.001)$ as compared to control or respective values for myasthenia gravis patients with no tumour. In the Hodgkin-type lymphoma the decrease in $\mathrm{H}$-subunit content was similar, but $\mathrm{LDH}_{4}$ was significantly increased $(30.4 \%, \mathrm{p}<$ $0 \cdot 001$ ) instead of $\mathrm{LDH}_{5}$.

\section{Discussion}

According to Plum et $a l^{8}$ the intrathymic maturation of lymphocytes (when the bone marrow derived thymocyte precursors develop into mature immunocompetent T-cells) is associated with a steady increase in $\mathrm{LDH}_{1}$ relative activity, that is $\mathrm{H}$-subunit content. Since the activity of $\mathrm{LDH}_{1}$ is apparent in T-lymphocytes of peripheral blood and B-cells lack this isoenzyme, $\mathbf{L D H}_{1}$ can be used as a T-cell marker. ${ }^{7 y}$ Similar findings have been reported by Dioguardi et al ${ }^{16}$ who compared LDH isoenzyme patterns in mature thymocytes from adults and in foetal thymocytes. Thus, the significant increase of $\mathrm{LDH}_{1}$ activity and the slight elevation in $\mathrm{H}$-subunit content observed in the thymus of myasthenia gravis patients can be accounted for by an enhanced ratio of the mature, more immunocompetent thymocytes. This view is in line with the observation by Palestro et $a l^{17}$ who showed an elevated ratio of T-cell population in myasthenia gravis patients using alphanaphtyl-acetate-esterase activity, another marker of $\mathrm{T}$-cells. In myasthenia gravis patients this phenomenon is connected with enhanced activity of thymosin alpha $_{1}$, a thymic hormone described by Goldstein ${ }^{18}$ which is produced in the epithelial cells and is known to exert a growth and differentiation promoting action on thymic cells. It is probable that the ratio of T-cells was even higher than one would calculate based on the increased ratio of $\mathrm{LDH}_{1}$, since owing to the immune responses taking place in the thymus of affected patients, B-cells are also accumulating ${ }^{1}$ and this might counteract the elevation of $\mathrm{LDH}_{1}{ }^{7}$ As a result of the two opposing events the thymus of the myasthenia gravis patients as a whole responds by an elevation of $\mathrm{LDH}$ isoenzymes characteristic of the mature differentiated lymphocytes. This is compatible with recent observations indicating that mature differentiated T-lymphocytes play a significant role in the pathogenesis of the disease despite the fact that the neuromuscular blockade in myasthenia gravis is caused by specific antibodies produced by B-cells. The immunoreactive and mitogen-induced responses of mature $T$-cells are more enhanced than those by less differentiated T-cells. ${ }^{20}$ The number of these activated $\mathrm{T}$-cells that have undergone blast transformation is appreciably elevated in the thymus of myasthenia gravis patients in connection with the autoimmune responses. ${ }^{2}$ A subpopulation of specially differentiated mature T-lymphocytes ( $T$ helper cells) is involved in the cooperative action between $\mathrm{T}$ - and B-lymphocytes indispensable for antibody production. ${ }^{3}$ Thus, the increased thymic $\mathrm{LDH}_{1}$ activity and $\mathrm{H}$-subunit content appear to indicate an elevated ratio of mature T-lymphocytes with enhanced immunoreactivity. At the same time the deleterious effects of these T-cells might persist even years after thymectomy owing to the much 
longer life span of T-cells than B-cells. ${ }^{4}$ Study of the clinical states also seems to suggest that the stage of maturation and differentiation of the thymus (at least in the long run) might influence the course of disease. The elevated ratio of $\mathrm{H}$-subunit observed in the glands removed indicating a predominance of mature thymocytes over B-lymphocytes, was correlated with a poor clinical condition at a mean time of 6.5 years after surgery. On the other hand the lower proportion of $\mathrm{H}$-subunits indicating less severe clinical manifestations was associated with a relative increase in the numbers of less differentiated thymocytes and B-cells. The view that after removal of thymus with hyperplasia and a predominance of B-cells, a better prognosis can be expected has been expressed although not generally accepted. ${ }^{21}$

In the knowledge of the subunit composition of $\mathrm{LDH}$ isoenzymes one can see that the $\mathrm{H}$-subunit content is more informative than $\mathrm{LDH}_{1}$ alone, because in addition to $\mathrm{LDH}_{1}\left(\mathrm{H}_{4}\right)$ it includes a considerable part of $\mathrm{LDH}_{2}\left(\mathrm{H}_{3} \mathrm{M}_{1}\right)$ as well. The question then arises as to why $\mathrm{LDH}_{1}$ and $\mathrm{LDH}_{2}$ together (that is $\mathrm{H}$-subunit content) are more informative than $\mathrm{LDH}_{1}$ alone with regard to the long-term prognosis of this disease. It appears that the question is answered by the peculiar LDH isoenzyme pattern in a certain type of thymus tumours, that is in thymomas with minimal lymphocytic reaction. This type of thymoma, in addition to the proliferating epithelial cells, contains only few lymphocytes in contrast to the other type in which lymphocytes predominate as revealed by light microscopy." The relative activity of $\mathrm{LDH}_{5}$ was increased in all of the three thymomas with enhanced lymphocytic reactions. This is to be expected because in tumours, as a result of tissue hypoxia there is a shift in LDH isoenzyme pattern with prominence of $\mathrm{M}$-subunit otherwise characteristic of anaerobic conditions. ${ }^{22}$ The predominance of $\mathrm{LDH}_{4}$ in the Hodgkin-type lymphoma structurally similar to foetal thymus ${ }^{23}$ tissue is not surprising, since this finding is generally characteristic of undifferentiated tissues such as tumours. ${ }^{24}$ On the other hand in the three other thymoma cases associated with minimal lymphocytic reactions no such alterations were seen. Even so, LDH isoenzyme pattern was shifted to the opposite direction; $\mathrm{LDH}_{2}$ alone was enhanced markedly with ensuing increment in $\mathrm{H}$-subunit content. This type of change is however, characteristic of tissues with enhanced oxidative activities. ${ }^{25}$ In this particular tumour LDH isoenzyme composition is determined by the epithelial cells' increased oxidative activity and not by their tumour characteristics. The special secretory function of thymic epithelial cells has been proved in turn by several investigators. ${ }^{181926}$ Other reports likewise suggest that thymic epithelial cells are more abundant in oxidative enzymes than thymocytes ${ }^{27} 28$ and the secretory activity of epithelial cells in thymoma is strikingly high. ${ }^{29}$ Thus, it is presumed that the elevation of $\mathrm{LDH}_{2}$ is linked with secretory activity since in other endocrine glands LDH isoenzyme pattern is also known to change in association with secretory function. In the thymus with no tumour, owing to the markedly lower count of epithelial cells, $\mathrm{LDH}_{2}$ was below detection limit. In the knowledge of the role of thymic epithelial cells presumably played in the pathogenesis of myasthenia gravis, it is not surprising that even several years after surgery the patients' clinical conditions closely correlated with $\mathrm{H}$-subunit contents characterising both lymphoid and epitheloid components of the thymus.

In summary, the study of $\mathrm{LDH}$ isoenzyme $(\mathrm{H}-$ subunit) pattern in addition to its theoretical considerations also appears to have practical importance. It provides an aid in assessing the stage of thymic differentiation and thereby the long-term prognosis of thymectomised patients. An elevated $\mathrm{H}$-subunit content over twofold $(61 \%)$ normal value seems to indicate a poor prognosis. This information may be helpful in selecting further therapeutic regimens (steroids, immunosuppression or plasmapheresis) for thymectomised patients who have shown insufficient improvement in their clinical condition.

\section{References}

Abdou NI, Lisak RP, Zweiman B, Abrahamsohn I, Penn AS. The thymus in myasthenia gravis. Evidence for altered cell populations. N Engl J Med $\stackrel{?}{?}$ 1974;291: 1271-5.

${ }^{2}$ Christensson B, Biberfeld P, Matell G, Smith CIE, Hammarström L. Immunological findings in thymic biopsies in myasthenia gravis: thymic immunohistology and mitogen reactivity. Ann NY Acad Sci 1981;377:818-20.

${ }^{3}$ Wekerle H, Hohlfeld R. Thymic myogenesis, T-lymphocytes and the pathogenesis of myasthenia gravis. Ann NY Acad Sci 1981;377:455-75.

${ }^{4}$ Berrih S, LeBrigand H, Levasseur Ph, Gaud C, Bach JF. Depletion of helper/inducer $\mathrm{T}$ cells after thymectomy in myasthenic patients. Clin Immunol Immunopathol 1983;28:272-81.

${ }^{5}$ Kulenkampff J, Janossy G, Greaves MF. Acid esterase in human lymphoid cells and leukaemic blasts: a marker for $\mathrm{T}$ lymphocytes. $\mathrm{Br} \mathrm{J}$ Haematol 1977;36:231-40.

- Bollum FJ. Terminal deoxynucleotidyl tranferase as a hematopoietic cell marker. Blood 1979;54:1203-15.

${ }^{7}$ Plum J, Ringoir S. Lactate dehydrogenase isoenzyme pattern as a measure of cellular differentiation in lymphocytic cells. J Rectic Soc 1977;21:225-30.

${ }^{8}$ Plum J, Ringoir S, de Smedt M. Lactic-dehydrogenase activity in thymocyte populations. Lancet $1977 ; 2: 721$. 
' Sabbe L, Plum J, de Smedt M, Leroux G, Louwagie A, Criel A. Cell-marker studies in CLL with monoclonal OKT antisera and lactic dehydrogenase isoenzyme analysis. Blut 193;46:261-70.

${ }^{10}$ Rovensky J, Lukac J, Zitman D, Vojtkova J, Tovarek J, Lokaj $\mathbf{J}$. Isoenzymes of lactate dehydrogenase in $T$ lymphocytes in systemic lupus erythematosus. Arthritis Rheum 1983;26:932-3.

"Lapis K, Szende B, Répássy G, et al. Morphologic and clinico-pathologic features of thymus tumours. Acta Morph Acad Sci Hung 1981;29:109-25.

${ }^{12}$ Wieme RJ. An improved technique of agar-gel electrophoresis on microscope slides. Clin Chim Acta $1959 ; 4: 317-21$

${ }^{13}$ Van der Helm RJ. Simple method of demonstrating lactic acid dehydrogenase isoenzymes. Lancet 1961;2:108-9.

${ }_{14}$ Thorling EB, Jensen K. The lactate dehydrogenase isoenzymes in various organs of the rabbit in anaemia, hypoxia, and after cobalt administration. Acta Pathol Microbiol Scand 1966;66:426-36.

is Szobor A. Myasthenia gravis: a quantitative evaluation system. Disability Status Scale (DSS) applied for myasthenia gravis. Eur Neurol 1976;14:439-46.

${ }^{16}$ Dioguardi N, Ideo G, Mannucci PM, Piorelli G. Lactate dehydrogenase (LDH), glutamic oxalacetic transaminase (GOT) and malate dehydrogenase (MDH) isoenzymes in lymphocytes from foetal and adult thymus, spleen and from peripheral blood. Enzym Biol Clin 1966;6:324-38.

${ }^{17}$ Palestro G, Valente G, Micca FB, Novero D, Arisio R. Detection and distribution of alfa-naphthyl acetate esterase activity in thymocytes of normal, myasthenic thymus and thymoma. Virchows Arch B Cell Path $1980 ; 35: 33-43$.

${ }^{18}$ Goldstein AL, Thurman GB, Low TLK, Rossio JL, Trivers GE. Hormonal influences on the reticuloendothelial system: current status of the role of thymosin in the regulation and modulation of immunity. $J$ Reticuloendothel Soc 1978;23:253-66.

${ }^{19}$ Dalakas MC, Engel WK, McClure JE, Goldstein AL,
Askanas V. Identification of human thymic epithelial cells with antibodies to thymosin $\alpha_{1}$ in myasthenia gravis. Ann NY Acad Sci 1981;377:477-84.

${ }^{20}$ Griend RJ van de, Astaldi GCB, Ende A van den, Loos JA, Roos D. Subpopulations of T lymphocytes from human blood differing in density and stage of maturation. Eur J Immunol 1980; 10:70-3.

${ }^{21}$ Penn AS, Jaretzki III A, Wolff M, Chang HW, Tennyson V. Thymic abnormalities: antigen or antibody? response to thymectomy in myasthenia gravis. Ann NY Acad Sci 1981;377:786-804.

${ }^{22}$ Goldman RD, Kaplan NO, Hall TC. Lactic dehydrogenase in human neoplastic tissues. Cancer Res 1964;24:389-99.

${ }^{23}$ Pfleiderer G, Wachsmuth ED. Alters- und funktionsabhängige Differenzierung der Lactatdehydrogenase menschlicher Organe. Bioch J 1961;334:185-98.

${ }^{24}$ Schapira F. Resurgence of fetal isozymes in cancer: study of aldolase, pyruvate kinase, Lactid dehydrogenase, and B-hexosaminidase. In: Isozymes: Current Topics in Biological and Medical Research Vol. 5. New York: Alan R Liss, Inc., 1981:27-75.

${ }^{25}$ Cahn RD, Kaplan NO, Levine L, Zwilling E. Nature and development of lactic dehydrogenases. The two major types of this enzyme form molecular hybrids which change in makeup during development. Science 1962;136: 962-69.

${ }^{26}$ White $\mathrm{A}$, Goldstein $\mathrm{AL}$. Is the thymus an endocrine gland? Old problem, new data. Perspect Biol Med 1968;11:475-89.

${ }^{27}$ Timperley WR, Ahmed A. Histochemistry of the thymus and a thymoma. Arch Pathol 1970;89:405-9.

${ }^{28}$ Timperley WR, Barson AJ, Davies S. Enzyme histochemistry of the developing human thymus. Biol Neonate 1971;19:42-54.

${ }^{29}$ Szekamova Sz, Répássy G, Lapis K, Szobor A, Szathmáry I. The fine structure of the thymus and neuromuscular junction in myasthenic and nonmyasthenic patients (in Hungarian). Morph és Ig Orv Szemle 1978;18:107-16. 